\title{
(2) OPEN ACCESS \\ Genetic aspects of idiopathic paediatric uveitis and juvenile idiopathic arthritis associated uveitis in Chinese Han
}

\author{
Jing Deng, ${ }^{1}$ Handan Tan, ${ }^{1}$ Jiayue Hu, ${ }^{1}$ Guannan Su, ${ }^{1}$ Qingfeng Cao, ${ }^{1}$ Xinyue Huang, \\ Chunjiang Zhou, ${ }^{1}$ Yao Wang, ${ }^{1}$ Aize Kijlstra, ${ }^{2}$ Peizeng Yang $\odot$
}

\begin{abstract}
- Additional material is
published online only. To view please visit the journal online (http://dx.doi.org/10.1136/ bjophthalmol-2018-313200).

${ }^{1}$ The First Affiliated Hospital of Chongqing Medical University, Chongqing Key Laboratory of Ophthalmology and Chongqing Eye Institute, Chongqing, P. R. China

${ }^{2}$ University Eye Clinic Maastricht, Maastricht, The Netherlands
\end{abstract}

Correspondence to Dr Peizeng Yang, The First Affiliated Hospital of Chongqing Medical University, Chongging 400016, China; peizengycmu@ 126.com

JD, HT and JH contributed equally.

Received 14 September 2018 Revised 11 February 2019 Accepted 9 March 2019 Published Online First 2 April 2019
Check for updates

(C) Author(s) (or their employer(s)) 2020. Re-use permitted under CC BY-NC. No commercial re-use. See rights and permissions. Published by BMJ.

To cite: Deng J, Tan H, Hu J, et al. Br J Ophthalmol

2020;104:443-447.

\section{ABSTRACT}

Background Idiopathic paediatric uveitis (IPU) and juvenile idiopathic arthritis associated uveitis (JIA-U) are the two most common entities in paediatric uveitis. This study addressed the possible association of IPU and JIA-U with genes that had been shown earlier to be associated with juvenile idiopathic arthritis.

Methods We carried out a case-control association study involving 286 IPU, 134 JIA-U patients and 743 healthy individuals. A total of 84 candidate single nucleotide polymorphisms (SNPs) in 60 genes were selected for this study. The MassARRAY platform and iPLEX Gold Genotyping Assay was used to genotype 83 candidate SNPs and the remaining SNP (rs27293) was analysed using the TaqMan SNP Genotyping Assay. Results No evidence was found for an association of the candidate polymorphisms tested with IPU. Six SNPs (PRM1/rs11074967, JAZF1/rs73300638, IRF5/ rs2004640, MEFV/rs224217, PSMA3/rs2348071 and PTPN2/rs7234029) showed an association with JIA-U $\left(p<1.0 \times 10^{-2}\right)$.

Conclusion Our findings showed associations of six SNPs (PRM1/rs11074967, JAZF1/rs73300638, IRF5/ rs2004640, MEFV/rs224217, PSMA3/rs2348071 and PTPN2/rs7234029) with JIA-U. No association was detected between the 84 tested SNPs and IPU.

\section{INTRODUCTION}

Uveitis is an intraocular inflammatory disease characterized by a complex aetiology and a high incidence of blindness. ${ }^{1}$ The proportion of children visiting a uveitis centre has been estimated to range between $2.2 \%$ and $13.8 \% .^{2-4}$ The intraocular inflammatory disease that occurs in children under the age of 16 is called paediatric uveitis (PU) and includes almost all clinical entities that also occur in adults. Uveitis in children is still a challenge to the treating ophthalmologist, owing to its complicated management and up to $25 \%-33 \%$ of children with uveitis lose sight due to inaccurate diagnosis and delayed treatment. ${ }^{5}$

In PU, non-infectious uveitis and bilateral anterior uveitis is the major subtypes and similar frequencies have been reported from different geographical regions and ethnic backgrounds. ${ }^{6-9}$ Only a few PU entities have been classified, including juvenile idiopathic arthritis (JIA), Behcet's disease (BD), Vogt-Koyanagi-Harada (VKH) disease and tubulointerstitial nephritis and uveitis syndrome. ${ }^{9-11}$ In particular, JIA is the most common systemic disorder underlying PU, accounting for 4\%-24\% of total PU. ${ }^{12-14}$ PU, whereby a clear aetiology cannot be established, is classified as idiopathic paediatric uveitis (IPU) and the frequency of this entity is similar to juvenile idiopathic arthritis associated uveitis (JIA-U). ${ }^{6-9}$

A genetic component is currently considered to play an important role in the pathogenesis of autoimmune disease. ${ }^{1516}$ Unravelling the genetic aspects of autoimmune disease may lead to the identification of the involved immune and inflammatory pathways and can result in novel treatment modalities. The possible genetic basis of IPU has not yet been widely studied and was therefore the subject of the study reported here. We focused on those genes which were previously shown to be significantly associated with JIA. Besides a group having IPU, we therefore also included JIA-U patients as a positive control.

\section{METHODS}

\section{Participants}

For this project, we recruited 286 IPU, 134 JIA-U patients and 743 controls at the First Affiliated Hospital of Chongqing Medical University from 2008 to 2017. All subjects were Han Chinese. Patients with IPU were younger than 16 years. We excluded a total amount of 188 patients with uveitis under the age of 16 with a clear aetiology, of which 72 had VKH disease, 18 had BD, 15 had Fuchs and 83 included minor uveitis entities. Patients with JIA-U were diagnosed following the International League of Associations for Rheumatology criteria. ${ }^{17}$ The 743 controls were matched for gender and geographic origin.

\section{Single nucleotide polymorphism choice}

Selection of single nucleotide polymorphisms (SNPs) was based on previously reported susceptibility loci in JIA with a P value less than $1.0 \times 10^{-2}$ by doing a PubMed search from inception to 2017. Based on the National Center of Biotechnology Information database in the Chinese Han population (CHB), candidate SNPs were checked by Haploview 4. The $\mathrm{r}^{2}$ value for linkage disequilibrium had to be less than 0.8 between adjacent loci and the minor allele frequency had to be larger than 0.05 in the CHB. Based on the above criteria, we finally included 84 SNPs in or near 60 loci: ANTXR2, ${ }^{18}$ CXCR $4,{ }^{18}$ CD226, ${ }^{19}$ TNFA $^{19}{ }^{19} \mathrm{MIF}^{19}$ CD28, ${ }^{19} \mathrm{KIAA}^{1109},{ }^{19}$ PSMC6, ${ }^{20}$ PSMA3 $^{20}{ }^{2}$ STAT4 $^{21}{ }^{2}$ PTPN2, ${ }^{21}$ IL21/ 
IL $2,{ }^{21}$ ERAP2/LNPEP, ${ }^{21}$ ERAP2/LNPEP, ${ }^{21}$ UBE2L $3,{ }^{21}$ C5 orf56/ IRF1, ${ }^{21}$ RUNX1, ${ }^{21}$ IL2RB, ${ }^{21}$ FAS, ${ }^{21}$ ZFP36L1, ${ }^{21}$ LTBR, ${ }^{21}$ COG6,${ }^{21}$ Chr13q14, ${ }^{21}$ PRR5L,${ }^{21}$ PRM1, ${ }^{21}$ RUNX3,${ }^{21}$ JAZF1, ${ }^{21}$ AFF3/LONRF2, ${ }^{21}$ FCRL3, ${ }^{22}$ TRAF5, ${ }^{23}$ MAPKAPK2, ${ }^{24}$ C1orf116, ${ }^{24}$ IL20, ${ }^{24}$ CDGAP/ARHGAP31, ${ }^{25}$ C3orf1/ TIMMDC1, ${ }^{25}$ IL15, ${ }^{25}$ NRBF2-EGR2, ${ }^{25}$ JMJD1C, ${ }^{25}$ REEP3, ${ }^{25}$ IGF1R-FAM169B, ${ }^{25}$ CHD9-TOX $3,{ }^{25}$ IRF $5,{ }^{26} \mathrm{BLK},{ }^{27} \mathrm{C} 5$ orf $30,{ }^{27}$ CD247, ${ }^{27}$ ORMDL $3,{ }^{27}$ PTPRC, ${ }^{27}$ ERAP1, ${ }^{28}$ LPP $^{29}$ TRAF1/C5, ${ }^{30}$ IL2RA, ${ }^{31}$ Intergenic, ${ }^{31}{ }^{32}$ ANGPT1, ${ }^{31}$ TMEM39A, ${ }^{31}$ PTPN2, ${ }^{31}$ ADAD1, ${ }^{31}$ VTCN1, ${ }^{32}$ MEFV, $^{33}$ IL1A, ${ }^{34}$ IL1RN, ${ }^{34}$ SLC26A2 ${ }^{35}$ (see online supplementary table 1).

\section{DNA extraction and genotyping}

Venous blood samples from all subjects were collected into EDTA containing tubes and stored at $-80^{\circ} \mathrm{C}$. Genomic DNA was extracted with the QIAamp DNA Blood Mini Kit (Qiagen, Valencia, California, USA) and stored at $-20^{\circ} \mathrm{C}$ until used. The Nanodrop 2000 (Thermo Fisher Scientific, Wilmington, Delaware, USA) was used to qualify and quantify all DNA samples. The primers for respective SNPs were designed by MassArray Assay Design V.3.1 software (Sequenom) and stored at $-20^{\circ} \mathrm{C}$. The selected SNPs were genotyped by MassARRAY platform (Sequenom, San Diego, California, USA) and iPLEX Gold Genotyping Assay, and analysed by TYPER software V.4.0. The PCR was performed with the GeneAmp PCR System 9700 thermocycler (ABI, Foster City, California, USA). Because none of the primers were suitable for Sequenom MassArray, the rs27293 genotype was analysed using the TaqMan SNP Genotyping Assay (ABI, Foster City, California, USA) with the 7500 RealTime PCR system (Applied Biosystems), and counted by TaqMan Genotyper Software. All assays were conducted in strict accordance with manufacturer's instructions. A 90\% SNP genotyping success rate was confirmed for all tested SNPs.

\section{Statistical analysis}

The $\chi 2$ test was used to analyse the Hardy-Weinberg equilibrium with SPSS (V.19.0). Genotype as well as allele frequencies between patient and control groups were compared using the $\chi 2$ test. ORs and 95\% CIs were calculated by SPSS as well. The Bonferroni correction method was used to adjust $p$ values to corrected $\mathrm{P}(\mathrm{Pc})$ according to the number of performed analyses for multiple comparisons. A Pc $<0.05$ was taken as significant.

\section{RESULTS}

\section{Clinical features of enrolled IPU and JIA-U patients}

The clinical features as well as demographic information of the tested IPU cases, JIA-U cases and healthy controls are shown in table 1 . The clinical characteristics of the IPU and JIA-U patients included age at disease onset, sex, band keratopathy, iridocyclitis, complicated cataract and arthritis. The clinical ocular characteristics of both entities is quite similar and the only significant difference is the presence of arthritis in the JIA-U group.

\section{Genotype and allele frequencies of examined SNPs in cases and controls}

Eighty-four gene loci were genotyped in this experiment. A total of 286 children with IPU, 134 with JIA-U and 743 controls were enrolled. No association was detected between the 84 candidate SNPs and IPU (online supplementary table 2). In the JIA-U group a significant association was observed for six SNPs (PRM1/rs11074967， JAZF1/rs73300638， IRF5/rs2004640, MEFV/rs224217, PSMA3/rs2348071 and PTPN2/rs7234029),
Table 1 Clinical characteristics, gender and testing age distribution of patients and controls

\begin{tabular}{|llc}
\hline & Total & $\%$ \\
\hline Patients with IPU & 286 & \\
\hline Mean age \pm SD & $9.6 \pm 3.6$ & 40.6 \\
\hline Male & 116 & 59.4 \\
\hline Female & 170 & 100 \\
\hline Iridocyclitis & 286 & 68.5 \\
\hline Band keratopathy & 196 & 50.3 \\
\hline Complicated cataract & 144 & 0 \\
\hline Arthritis & 0 & \\
\hline Patients with JIA-U & 134 & 37.3 \\
\hline Mean age \pm SD & $16.5 \pm 11.6$ & 62.7 \\
\hline Male & 50 & 100 \\
\hline Female & 84 & 56.0 \\
\hline Iridocyclitis & 134 & 69.4 \\
\hline Band keratopathy & 75 & 100 \\
\hline Complicated cataract & 93 & \\
\hline Arthritis & 134 & 40.8 \\
\hline Healthy controls & 743 & \\
\hline Mean age \pm SD & $39.0 \pm 11.7$ & \\
\hline Male & 440 & \\
\hline Female & 303 & \\
\hline
\end{tabular}

IPU, idiopathic paediatric uveitis; JIA-U, juvenile idiopathic arthritis associated uveitis.

respectively (table 2). No significant association was found for the other 78 SNPs and JIA-U (online supplementary table 3).

A significantly increased frequency of the CC genotype and C allele of PRM1/rs11074967 was detected in patients with JIA-U when compared with controls $\left(\mathrm{Pc}=2.39 \times 10^{-2}\right.$, $\mathrm{OR}=2.167$ and $\mathrm{Pc}=4.17 \times 10^{-2}, \mathrm{OR}=1.864$, respectively). $\mathrm{A}$ significant decrease was found in the frequency of the JAZF1/ rs73300638 AA genotype and A allele $\left(\mathrm{Pc}_{\mathrm{c}}=7.21 \times 10^{-3}\right.$, $\mathrm{OR}=0.420$ and $\mathrm{Pc}=2.84 \times 10^{-3}, \mathrm{OR}=0.564$, respectively), IRF5/rs2004640 GG genotype and G allele $\left(\mathrm{Pc}=6.07 \times 10^{-3}\right.$, $\mathrm{OR}=0.448$ and $\mathrm{Pc}=5.33 \times 10^{-3}, \mathrm{OR}=0.559$, respectively), $\mathrm{MEFV} / \mathrm{rs} 224217 \mathrm{GG}$ genotype and $\mathrm{G}$ allele $\left(\mathrm{Pc}=2.61 \times 10^{-2}\right.$, $\mathrm{OR}=0.483$ and $\mathrm{Pc}=3.39 \times 10^{-2}, \mathrm{OR}=0.572$, respectively), PSMA3/rs2348071 AA genotype and A allele $\left(\mathrm{Pc}=2.53 \times 10^{-2}\right.$, $\mathrm{OR}=0.405$ and $\mathrm{Pc}=9.40 \times 10^{-3}, \mathrm{OR}=0.586$, respectively) and PTPN2/rs7234029 AA genotype and A allele $\left(\mathrm{Pc}=5.52 \times 10^{-3}\right.$, $\mathrm{OR}=0.426$ and $\mathrm{Pc}=1.16 \times 10^{-2}, \mathrm{OR}=0.586$, respectively) in JIA patients with uveitis as compared with healthy controls (table 2) . Pooling of the two patient groups resulted in a lower risk association for PRM1/rs11074967, IRF5/rs2004640, $\mathrm{MEFV} / \mathrm{rs} 224217, \quad$ PSM3/rs2348071, PTPN2/rs7234029 compared with to the JIAU population alone, whereas in case of JAZF/rs73300638, a more significant Pc value was found in the combined JIA-U and IPU groups (online supplementary table 4).

\section{DISCUSSION}

In the present study, we investigated the association between 84 candidate SNPs with IPU and JIA-U in a Chinese Han population, whereby SNPs were selected on the basis that they had been reported earlier to be associated with JIA. No associations were found in the IPU group, whereas we were able to confirm an association between six candidate SNPs (PRM1/rs11074967, JAZF1/ rs73300638, IRF5/rs2004640, MEFV/rs224217, PSMA3/ rs2348071 and PTPN2/rs7234029) with JIA-U. ${ }^{2021263133}$ To the 
Table 2 Genotype and allele frequencies of PRM1, JAZF1, IRF5, MEFV, PSMA3 and PTPN2 polymorphisms in patients with JIA-U versus healthy controls

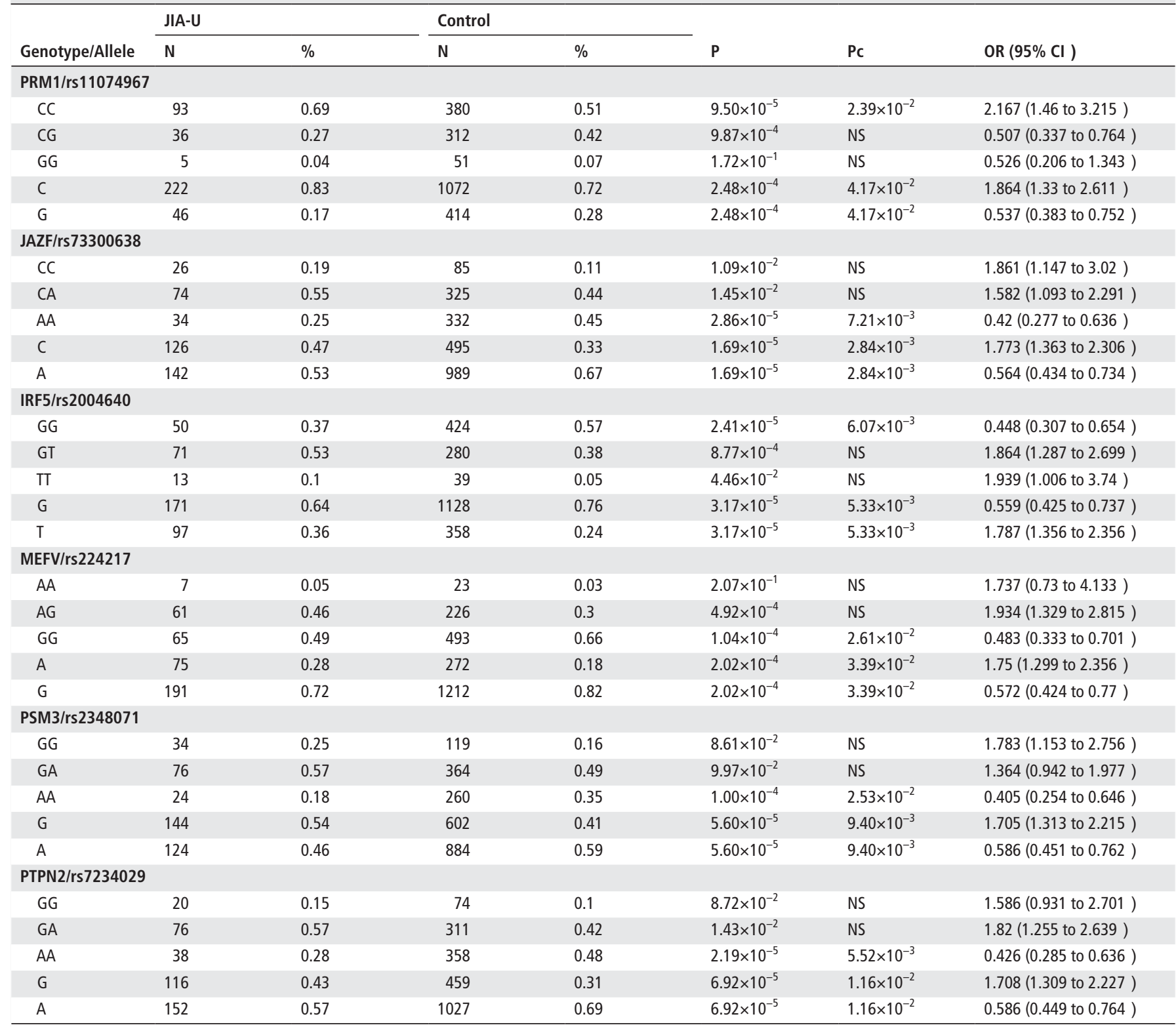

JIA-U, juvenile idiopathic arthritis associated uveitis; NS, not significant; Pc, Bonferroni corrected $p$ value.

best of our knowledge, this is the first report that addressed the issue whether JIA-U and IPU have a shared genetic background.

Not many studies have addressed the genetic aspects of PU. A significant association has been reported between PU and gene polymorphisms in MiR-146a (MicroRNA) and Ets-1 (Ets gene family of transcription factor. ${ }^{36}$ Earlier studies showed an association between rs12569232 and rs6540679 of TRAF5 with PU. ${ }^{23}$ However, these studies enrolled patients without excluding patients with JIA or any form of arthritis. According to the clinical manifestations of patients in our study, we found that IPU and JIA-U are very similar. The distinction between the two diseases mainly depends on whether patients have idiopathic arthritis. Furthermore, our findings show that the genetic basis of IPU and JIA-U may be quite different, which supports the concept that the two diseases should be considered as two separate clinical entities.

JIA is considered as the most common inflammatory rheumatic disorder in childhood, and is defined as a group of unexplained chronic joint disorders, which occur before the 16 th year of life and lasting for a time period of at least 6 weeks. ${ }^{17}$ In the recent decade, genome-wide association studies (GWAS) as well as exome sequencing and SNP analysis have identified a number of immune response pathways that are involved in the development of JIA. A GWAS study involving 2816 JIA individual and 13056 healthy controls confirmed association of three known JIA risk loci (the human leucocyte antigen region, PTPN22 and PTPN2) and identified 14 new loci by immunochip analysis. ${ }^{21}$ A genetic association study was also performed in patients from Northwest European descent and confirmed the association of several genes (PTPN22, VTCN1, the IL2-IL21 region, ANKRD55 and TNFA) with JIA. ${ }^{19}$

We confirmed the JIA-U susceptibility locus in rs11074967, which is located near the PRM1 gene. PRM1 is a member of protamines which have been isolated from spermatozoa, playing a role in sperm chromatin condensation. ${ }^{38}$ Most studies on PRM1 are related to male infertility. ${ }^{3940}$ The association between JIA and PRM1/rs11074967 was discovered using GWAS and it was suggested that the G allele of PRM1/rs11074967 was a 
protective factor, which is in agreement with our findings. This locus is novel and has not been found to be relevant in other diseases, and its function is still unclear.

The association of JIA with JAZF1/rs73300638, was also reported by this group. ${ }^{21}$ The rs73300638 polymorphism is on the intron of the gene that encodes the protein named 'juxtaposed with another zinc finger protein 1' (JAZF1). In agreement with the earlier GWAS study in JIA, ${ }^{21}$ we also found that the C allele of rs73300638 acts as a risk variant for JIA-U. This variant was not found to be associated with other diseases. The biological function of this locus is unknown. SNPs in JAZF were also shown to be associated with type 2 diabetes (T2DM) susceptibility, in subjects belonging to various different ethnic populations. ${ }^{41-43}$

Interferon regulatory factor 5 (IRF5) is a transcription factor that acts as repressor or activator of type I interferon genes and plays an important role in the induction of several proinflammatory cytokines. ${ }^{44} 45$ Associations have been reported for the IRF5 gene with immune disorders such as JIA, rheumatoid arthritis (RA), systemic lupus erythematosus and systemic sclerosis (SSc). ${ }^{26}{ }^{46-48}$ IRF5/rs2004640 is located in the first exon of IRF5 and it was reported that patients with JIA, SLE, SSc or RA have a higher frequency of the T allele and TT genotype of rs2004640 compared with controls. ${ }^{2647-49}$ Although our data failed to demonstrate a significant increase in the TT genotype of rs2004640 in JIA-U patients, we did find a significantly higher frequency of the $\mathrm{T}$ allele in JIA-U patients and this result was consistent with data found in the other diseases mentioned above.

The MEFV gene encodes a protein called pyrin (also known as marenostrin) that controls caspase- 1 activation and IL- $1 \beta$ processing. ${ }^{50}$ We hypothesise that polymorphisms of MEFV may affect IL-1 $\beta$ production and processing, which in turn may predispose to JIA-U although further experiments are needed to prove this assumption. Our results are consistent with earlier reports in JIA, ${ }^{33}$ showing that the G allele and GG genotype of rs224217 act as protective factors. This variant was also investigated in inflammatory bowel disease ${ }^{51}$ and $\mathrm{BD},{ }^{52}$ but no significant associations were found. Other variants of MEFV (rs224224 and rs224223) have been shown to be associated with familial Mediterranean fever. ${ }^{53}$

The proteasome subunit alpha type-3 (PSMA3) gene encodes a protein named macropain subunit $\mathrm{C} 8 .{ }^{54}$ Being a component of the ubiquitin-proteasome system, genetic variations in PSMA3 have been identified as susceptibility factors for several autoimmune diseases such as $\mathrm{JIA}^{20}$ and type 1 diabetes mellitus (T1DM). ${ }^{54}$ Previous studies have only found that the AG genotype of PSMA3/ rs2348071 is a risk genotype of JIA, but our study failed to reach the same conclusion. Our data showed a significantly decrease frequency of the G allele and GG genotype of rs2348071 in patients with JIA-U. The reason for this discrepancy is not yet clear. Like many of the other disease associated loci, the biological function of this polymorphism is also not yet known.

PTPN2 acts as a key regulatory factor in the T-cell-mediated immune response via Janus kinase/signal transducers and activators of the JAK/STAT pathway. ${ }^{55}$ The A allele and AA genotype of PTPN2/rs7234019 was also identified to be a protective factor for JIA by the GWAS study mentioned above ${ }^{21}$ and was now confirmed in our study in JIA-U. A polymorphism of PTPN2 was reported earlier by our group to be associated with uveitis in patients with BD. ${ }^{56}$ In that study we showed that carriers of the AA and AG genotypes in rs7234029 showed a significantly lower mRNA expression of PTPN2 than those carrying the GG genotype. ${ }^{56}$ These findings suggested that a variant of rs7234029 may affect the JAK1/STAT pathway and may contribute to the inflammation seen in JIA and JIA-U patients.

Our study has several limitations which we would like to mention here. We only enrolled Chinese Han patients and our data need to be confirmed in patients from other ethnic backgrounds. Furthermore, we only included JIA patients with uveitis and it would be interesting to see whether the same set of SNPs shows similar results in a Han Chinese JIA population without uveitis. It is possible that the associations found are related to the joint and not to the intraocular manifestations. It is also possible that genetic susceptibility is related to early onset, oligoarthritis and ANA status. Our sample size is however too small to perform a reliable analysis in separate subgroups. We have also not been able to collect enough Chinese JIA patients without uveitis and have not been able to test the gene associations in this separate patient group and hope to address this issue in the future. Our data should also not be extrapolated to other autoimmune or autoinflammatory uveitis entities. Additionally, it should be realised that IPU may not be a distinct clinical entity and could be a gathering of different intraocular inflammatory diseases in children, which may obscure potential associations with gene polymorphisms of the various immune response and inflammation pathways. Careful clinical descriptions and regular follow-up of this patient group is needed to resolve this issue. Another limitation is the fact that it is not yet exactly known whether some of the variants identified are functional variants and even if this would be known, how this would exactly affect the pathogenesis of uveitis in children with JIA. Moreover, since JIA comprises a heterogeneous group of chronic arthritis, which can be divided into seven distinct subtypes, based on clinical characteristics and laboratory parameters, there are some drawbacks of our study concerning the assumption that our disease control population is genetically homogenous. A larger patient sample size might allow the comparison with a phenotypically more homogeneous patient group.

In summary, our findings showed no evidence for an association of JIA related gene polymorphisms with IPU in Han Chinese, which lends support to the fact that one is clearly dealing with two different uveitis entities, with a similar clinical appearance but each with its own prognosis and clinical management.

Contributors PY and JH conceived and designed the study. JD, HT, JH, QC, XH, CZ and $Y W$ prepared the samples, materials and performed the experiments. JD, JH and HT wrote the initial draft of the paper. GS, AK and PY reviewed the data analysis and edited the manuscript. All authors read and approved the manuscript.

Funding This work was supported by Natural Science Foundation Major International (Regional) Joint Research Project grant number 81720108009 Chongqing Key Laboratory of Ophthalmology grant number CSTC, 2008CA5003, and the Natural Science Foundation Project of Chongqing grant number cstc2017shmsA130073. Thanks to all donors enrolled in the present study.

Competing interests None declared.

Patient consent for publication Before participant recruitment, every investigated individual was fully informed about the study and signed an informed consent. Informed consent from the patients who were younger than 18 years was obtained from their parents or guardians.

Ethics approval Experimental procedures and research design were conducted in accordance with the tenets of the Declaration of Helsinki and received the approval from the Clinical Research Ethics Committee of the First Affiliated Hospital of Chongqing Medical University (permit No. 2009-201008).

Provenance and peer review Not commissioned; externally peer reviewed.

Data sharing statement All data relevant to the study are included in the article or uploaded as supplementary information.

Open access This is an open access article distributed in accordance with the Creative Commons Attribution Non Commercial (CC BY-NC 4.0) license, which permits others to distribute, remix, adapt, build upon this work non-commercially, 
and license their derivative works on different terms, provided the original work is properly cited, appropriate credit is given, any changes made indicated, and the use is non-commercial. See: http://creativecommons.org/licenses/by-nc/4.0/.

\section{ORCID iD}

Peizeng Yang http://orcid.org/0000-0002-2647-6619

\section{REFERENCES}

1 Yang $\mathrm{P}$, Zhang Z, Zhou $\mathrm{H}$, et al. Clinical patterns and characteristics of uveitis in a tertiary center for uveitis in China. Curr Eye Res 2005;30:943-8.

2 Acharya NR, Tham VM, Esterberg E, et al. Incidence and prevalence of uveitis: results from the Pacific ocular inflammation study. JAMA Ophthalmol 2013:131:1405-12.

3 Smith JA, Mackensen F, Sen HN, et al. Epidemiology and course of disease in childhood uveitis. Ophthalmology 2009;116:1551.e1:1544-51.

4 Gritz DC, Wong IG. Incidence and prevalence of uveitis in Northern California; the Northern California epidemiology of uveitis study. Ophthalmology 2004; 111:491-500.

5 Zierhut M, Michels $\mathrm{H}$, Stübiger $\mathrm{N}$, et al. Uveitis in children. Int Ophthalmol Clin 2005;45:135-56.

6 Al-Mendalawi MD. Demographic and clinical features of pediatric uveitis at a tertiary referral center in Iran. Middle East Afr J Ophthalmol 2017;24.

7 Keino $\mathrm{H}$, Watanabe T, Taki W, et al. Clinical features of uveitis in children and adolescents at a tertiary referral centre in Tokyo. Br J Ophthalmol 2017;101:406-10.

8 Gautam N, Singh R, Agarwal A, et al. Pattern of pediatric uveitis at a tertiary referral institute in North India. Ocul Immunol Inflamm 2018:26:379-85.

9 Dajee KP, Rossen JL, Bratton ML, et al. A 10-year review of pediatric uveitis at a Hispanic-dominated tertiary pediatric ophthalmic clinic. Clin Ophthalmol 2016;10:1607-12

10 Tugal-Tutkun I. Pediatric uveitis. J Ophthalmic Vis Res 2011:6:259-69.

11 Wentworth BA, Freitas-Neto CA, Foster CS. Management of pediatric uveitis. F1000Prime Rep 2014:6

12 Heiligenhaus A, Heinz C, Edelsten C, et al. Review for disease of the year: epidemiology of juvenile idiopathic arthritis and its associated uveitis: the probable risk factors. Ocul Immunol Inflamm 2013;21:180-91.

13 Carvounis PE, Herman DC, Cha S, et al. Incidence and outcomes of uveitis in juvenile rheumatoid arthritis, a synthesis of the literature. Graefes Arch Clin Exp Ophthalmol 2006:244:281-90.

14 Sen ES, Dick AD, Ramanan AV. Uveitis associated with juvenile idiopathic arthritis. Nat Rev Rheumato/ 2015;11:338-48.

15 Hou S, Du L, Lei B, et al. Genome-wide association analysis of Vogt-Koyanagi-Harada syndrome identifies two new susceptibility loci at 1p31.2 and 10q21.3. Nat Genet 2014:46:1007-11.

16 Du L, Kijlstra A, Yang P. Vogt-Koyanagi-Harada disease: novel insights into pathophysiology, diagnosis and treatment. Prog Retin Eye Res 2016;52:84-111.

17 Petty RE, Southwood TR, Manners P, et al. International League of associations for rheumatology classification of juvenile idiopathic arthritis: second revision, Edmonton, 2001. J Rheumatol 2004;31:390-2.

18 Finkel TH, Li J, Wei Z, et al. Variants in CXCR4 associate with juvenile idiopathic arthritis susceptibility. BMC Med Genet 2016;17.

19 Reinards THCM, Albers HM, Brinkman DMC, et al. CD226 (DNAM-1) is associated with susceptibility to juvenile idiopathic arthritis. Ann Rheum Dis 2015;74:2193-8.

20 Sjakste T, Paramonova N, Rumba-Rozenfelde I, et al. Juvenile idiopathic arthritis subtype- and sex-specific associations with genetic variants in the PSMA6/PSMC6/ PSMA3 gene cluster. Pediatr Neonatol 2014;55:393-403.

21 Hinks A, Cobb J, Marion MC, et al. Dense genotyping of immune-related disease regions identifies 14 new susceptibility loci for juvenile idiopathic arthritis. Nat Genet 2013:45:664-9.

22 Ramírez-Bello J, Jiménez-Morales S, Espinosa-Rosales F, et al. Juvenile rheumatoid arthritis and asthma, but not childhood-onset systemic lupus erythematosus are associated with FCRL3 polymorphisms in Mexicans. Mol Immunol 2013;53:374-8.

23 Xiang Q, Chen L, Fang J, et al. TNF receptor-associated factor 5 gene confers genetic predisposition to acute anterior uveitis and pediatric uveitis. Arthritis Res Ther 2013:15.

24 Omoyinmi E, Forabosco P, Hamaoui R, et al. Association of the IL-10 gene family locus on chromosome 1 with juvenile idiopathic arthritis (JIA). PLoS One 2012;7:e47673.

25 Thompson SD, Marion MC, Sudman M, et al. Genome-wide association analysis of juvenile idiopathic arthritis identifies a new susceptibility locus at chromosomal region 3q13. Arthritis Rheum 2012:64:2781-91.

26 Nordang GBN, Viken MK, Amundsen SS, et al. Interferon regulatory factor 5 gene polymorphism confers risk to several rheumatic diseases and correlates with expression of alternative thymic transcripts. Rheumatology 2012;51:619-26.

27 Hinks A, Cobb J, Sudman M, et al. Investigation of rheumatoid arthritis susceptibility loci in juvenile idiopathic arthritis confirms high degree of overlap. Ann Rheum Dis 2012;71:1117-21.
28 Hinks A, Martin P, Flynn E, et al. Subtype specific genetic associations for juvenile idiopathic arthritis: ERAP1 with the enthesitis related arthritis subtype and IL23R with juvenile psoriatic arthritis. Arthritis Res Ther 2011;13.

29 Hinks A, Martin P, Flynn E, et al. Investigation of type 1 diabetes and coeliac disease susceptibility loci for association with juvenile idiopathic arthritis. Ann Rheum Dis 2010;69:2169-72.

30 Hinks A, Eyre S, Ke X, et al. Overlap of disease susceptibility loci for rheumatoid arthritis and juvenile idiopathic arthritis. Ann Rheum Dis 2010:69:1049-53.

31 Thompson SD, Sudman M, Ramos PS, et al. The susceptibility loci juvenile idiopathic arthritis shares with other autoimmune diseases extend to PTPN2, COG6, and ANGPT1. Arthritis Rheum 2010;62:3265-76.

32 Hinks A, Barton A, Shephard N, et al. Identification of a novel susceptibility locus for juvenile idiopathic arthritis by genome-wide association analysis. Arthritis Rheum 2009;60:258-63.

33 Day TG, Ramanan AV, Hinks A, et al. Autoinflammatory genes and susceptibility to psoriatic juvenile idiopathic arthritis. Arthritis Rheum 2008;58:2142-6.

34 Stock CJW, Ogilvie EM, Samuel JM, et al. Comprehensive association study of genetic variants in the IL-1 gene family in systemic juvenile idiopathic arthritis. Genes Immun 2008:9:349-57.

35 Lamb R, Thomson W, Ogilvie EM, et al. Positive association of Slc26a2 gene polymorphisms with susceptibility to systemic-onset juvenile idiopathic arthritis. Arthritis Rheum 2007;56:1286-91.

36 Wei L, Zhou Q, Hou S, et al. MicroRNA-146a and Ets-1 gene polymorphisms are associated with pediatric uveitis. PLoS One 2014:9:e91199.

37 Berntson L, Andersson Gäre B, Fasth A, et al. Incidence of juvenile idiopathic arthritis in the Nordic countries. A population based study with special reference to the validity of the ILAR and EULAR criteria. J Rheumato/ 2003;30:2275-82.

38 Oliva R, Dixon GH. Vertebrate protamine genes and the histone-to-protamine replacement reaction. Prog Nucleic Acid Res Mol Biol 1991;40:25-94.

39 Okada Y, Scott G, Ray MK, et al. Histone demethylase JHDM2A is critical for TNP1 and Prm1 transcription and spermatogenesis. Nature 2007:450:119-23.

40 Cho C, Willis WD, Goulding EH, et al. Haploinsufficiency of protamine- 1 or -2 causes infertility in mice. Nat Genet 2001:28:82-6.

41 Saxena R, Voight BF, Lyssenko V, et al. Genome-wide association analysis identifies loci for type 2 diabetes and triglyceride levels. Science 2007;316:1331-6.

42 Lee $\mathrm{Y}-\mathrm{H}$, Kang ES, Kim SH, et al. Association between polymorphisms in SIc30a8, Hhex, CDKN2A/B, IGF2BP2, FTO, WFS1, CDKAL1, KCNQ1 and type 2 diabetes in the Korean population. J Hum Genet 2008;53:991-8.

$43 \mathrm{Hu}$ C, Zhang R, Wang C, et al. PPARG, KCNJ11, CDKAL1, CDKN2A-CDKN2B, IDEKIF11-HHEX, IGF2BP2 and SIC30a8 are associated with type 2 diabetes in a Chinese population. PLoS One 2009;4:e7643.

44 Takaoka A, Yanai H, Kondo S, et al. Integral role of IRF-5 in the gene induction programme activated by Toll-like receptors. Nature 2005:434:243-9.

45 O'Neill LAJ, Bowie AG. The family of five: TIR-domain-containing adaptors in Toll-like receptor signalling. Nat Rev Immunol 2007;7:353-64.

46 Shimane K, Kochi Y, Yamada R, et al. A single nucleotide polymorphism in the IRF5 promoter region is associated with susceptibility to rheumatoid arthritis in the Japanese population. Ann Rheum Dis 2009:68:377-83.

47 Hammad A, Mossad YM, Nasef N, et al. Interferon regulatory factor 5 gene polymorphism in Egyptian children with systemic lupus erythematosus. Lupus 2017;26:871-80.

48 Dieudé $\mathrm{P}$, Guedj M, Wipff J, et al. STAT4 is a genetic risk factor for systemic sclerosis having additive effects with IRF5 on disease susceptibility and related pulmonary fibrosis. Arthritis Rheum 2009:60:2472-9.

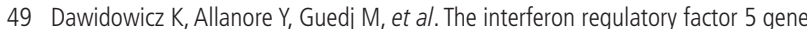
confers susceptibility to rheumatoid arthritis and influences its erosive phenotype. Ann Rheum Dis 2011;70:117-21.

50 Chae JJ, Wood G, Richard K, et al. The familial Mediterranean fever protein, pyrin, is cleaved by caspase- 1 and activates NF-kappaB through its N-terminal fragment. Blood 2008; 112:1794-803.

51 Villani A-C, Lemire M, Louis E, et al. Genetic variation in the familial Mediterranean fever gene (MEFV) and risk for Crohn's disease and ulcerative colitis. PLoS One 2009:4:e7154.

52 Jiang $\mathrm{Y}$, Wang $\mathrm{H}$, Yu $\mathrm{H}$, et al. Two genetic variations in the IRF8 region are associated with Behçet's disease in Han Chinese. Sci Rep 2016;6

53 Öksuz MF, Karkucak M, Görukmez O, et al. Investigation of MEFV gene polymorphisms ( $1138 \mathrm{G}$ and $\mathrm{A} 165 \mathrm{~A}$ ) in adult patients with familial Mediterranean fever. Rev Bras Reumatol Engl Ed 2017;57:501-6.

54 Sjakste T, Paramonova N, Osina K, et al. Genetic variations in the PSMA3, PSMA6 and PSMC6 genes are associated with type 1 diabetes in Latvians and with expression level of number of UPS-related and T1DM-susceptible genes in HapMap individuals. Mol Genet Genomics 2016;291:891-903.

55 Long SA, Cerosaletti K, Wan JY, et al. An autoimmune-associated variant in PTPN2 reveals an impairment of IL-2R signaling in CD4+ T cells. Genes Immun 2011:12:116-25.

56 Zhang Q, Li H, Hou S, et al. Association of genetic variations in PTPN2 and CD122 with ocular Behcet's disease. Br J Ophthalmol 2018;102:996-1002. 